\title{
Imaging Short-range Order and Extracting 3-D Strain Tensor Using Energy- filtered 4D-STEM Techniques
}

Ruopeng Zhang ${ }^{1}$, Steven Zeltmann ${ }^{1}$, Colin Ophus ${ }^{2}$, Benjamin Savitzky ${ }^{3}$, Thomas Pekin ${ }^{4}$, Eric Rothchild ${ }^{1}$, Karen Bustillo ${ }^{3}$, Mark Asta ${ }^{1}$, Daryl Chrzan ${ }^{1}$ and Andrew Minor ${ }^{1,2}$

${ }^{1}$ University of California-Berkeley, Berkeley, California, United States, ${ }^{2}$ Lawrence Berkeley National Lab, Berkeley, California, United States, ${ }^{3}$ National Center for Electron Microscopy (NCEM), Molecular Foundry, Lawrence Berkeley National Laboratory, Berkeley, California, United States, ${ }^{4}$ HumboldtUniversität zu Berlin, Berlin, Berlin, Germany

The existence of short-range order (SRO) has a significant impact on the mechanical properties in metallic alloys [1-3], where the deformation behaviors of the alloys can be directly correlated to the degree of SRO [4-6]. Recently, we have shown that the SRO domain structures can be directly imaged via Energy-filtered Transmission Electron Microscopy (EFTEM), in both Ti-Al alloys and CrCoNi medium entropy alloys $[7,8]$. With the presence of an SRO domain cluster, one of the hypothesized mechanisms to explain the SRO-induced strengthening is based on the potent interaction between SRO domain clusters and gliding dislocations, where leading dislocations need to overcome the energy barrier of creating "diffuse" antiphase boundaries (DAPB) to slip[9, 10]. A diffuse residual strain field would raise from the creation of DAPBs, altering following dislocation motions and local stacking fault energy. Direct measurement of the local strain of the DAPB is, therefore, of great interest for understanding the atomic origin of SRO strengthening and its relation to deformation behavior such as dislocation motion and deformation twinning.

With the adoption of Four-Dimensional Scanning Transmission Electron Microscopy (4D-STEM), strain mapping at nanometer spatial resolution is routinely reachable [11, 12]. Coupled with patterned beam forming apertures, the accuracy of the strain measurement from 4D-STEM could be significantly improved to $\sim 0.3 \%$ [13], enabling mapping of small strain variation from the DAPB. We developed a multi-zone 4D-STEM strain mapping technique to extract the full strain tensor of DAPB inside a region of material associated with planar slip. With the strain tensor determined, a direct correlation of strain to the local bonding environment becomes possible. Here we will present the first direct measurement of the strain tensor of a planar slip band (Figure 1) and relate it to computational simulations of DAPB and mechanical behavior in structural alloys, providing a new method to quantify the SRO-related deformations [14]. 

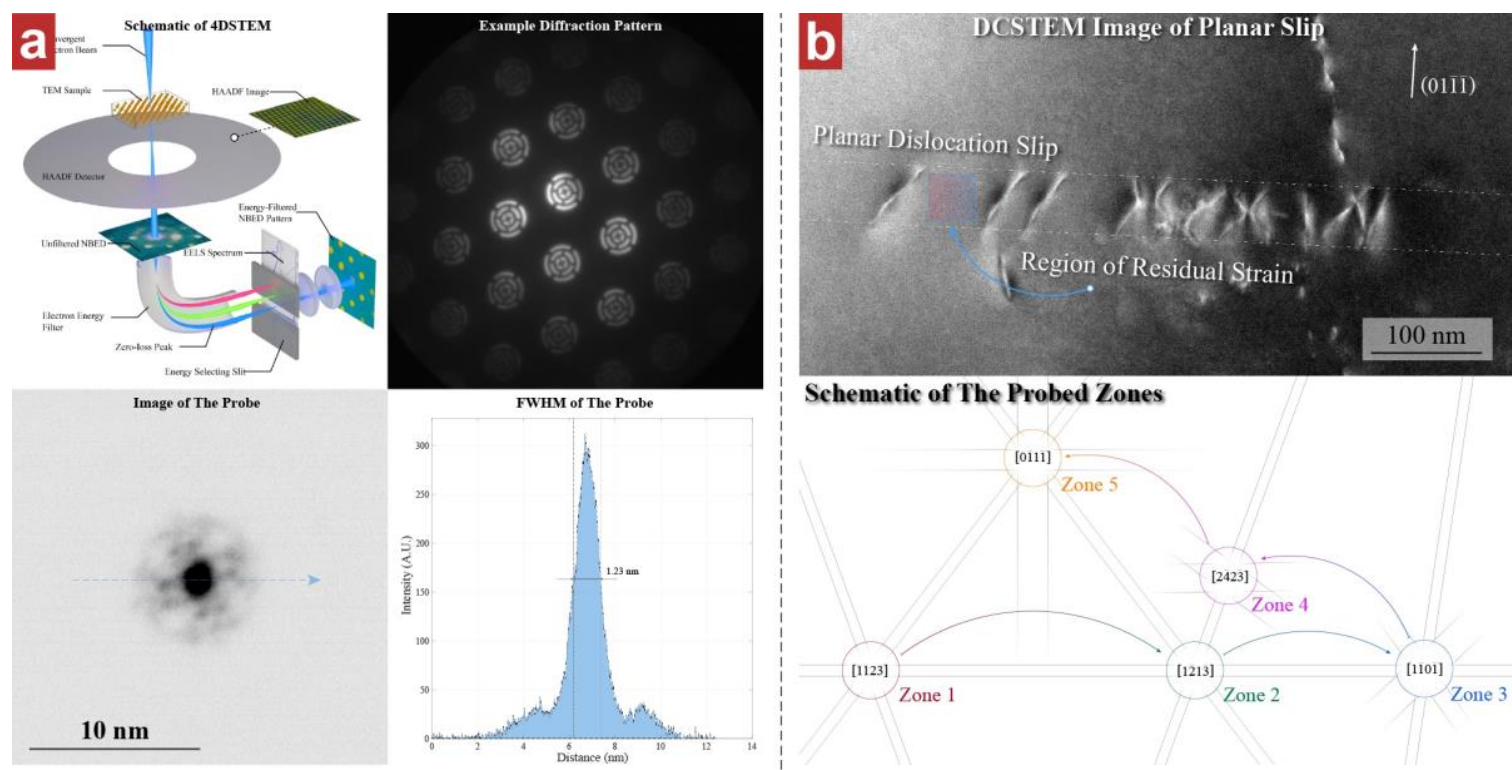

Schematic of The Probed Zones

Figure 1. Beam condition and schematics of the multi-zone 4D-STEM strain mapping of a planar slip band (DAPB); (a), The electron probe from the patterned beam forming aperture; (b), Diffraction contrast STEM image of the interested planar slip and the Kikuchi map showing the associated zone axes.
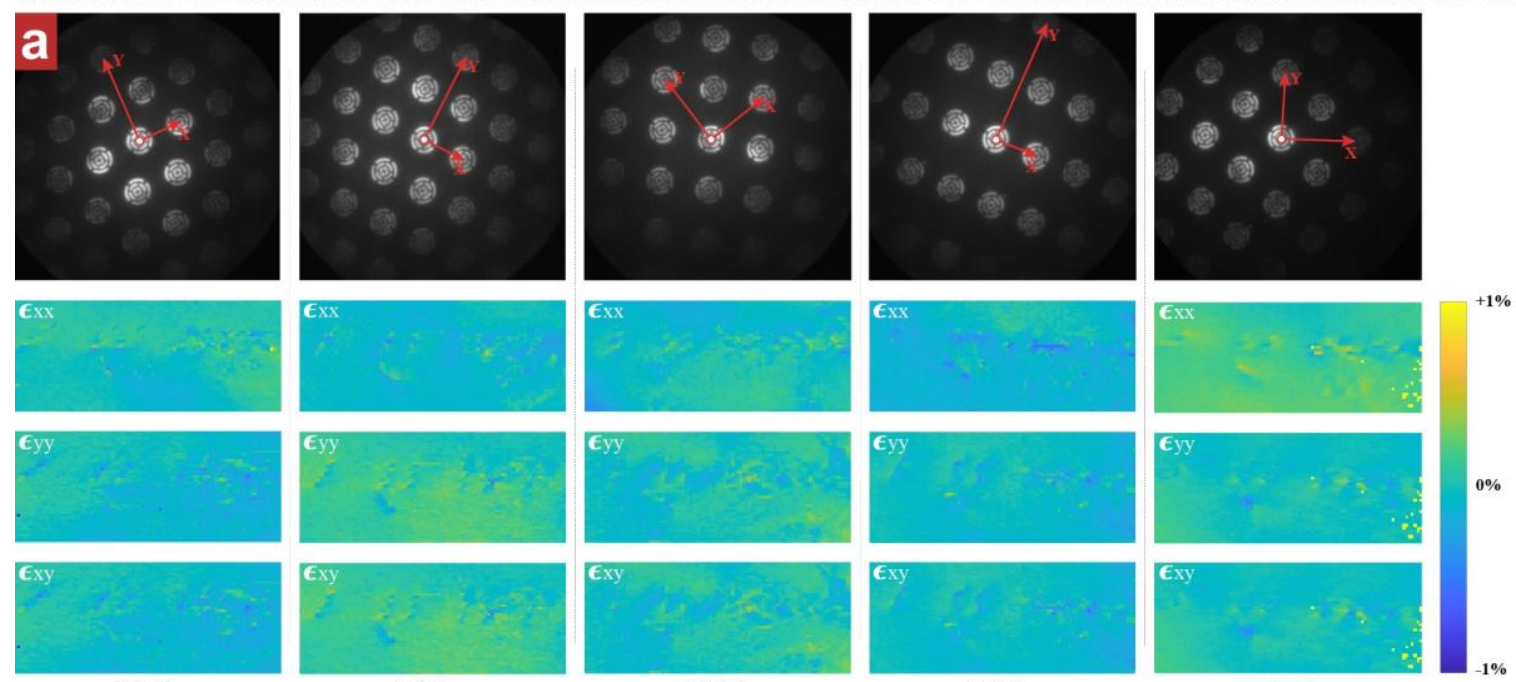

[1123]

[1213]

[1101]

[2423]

[0111]
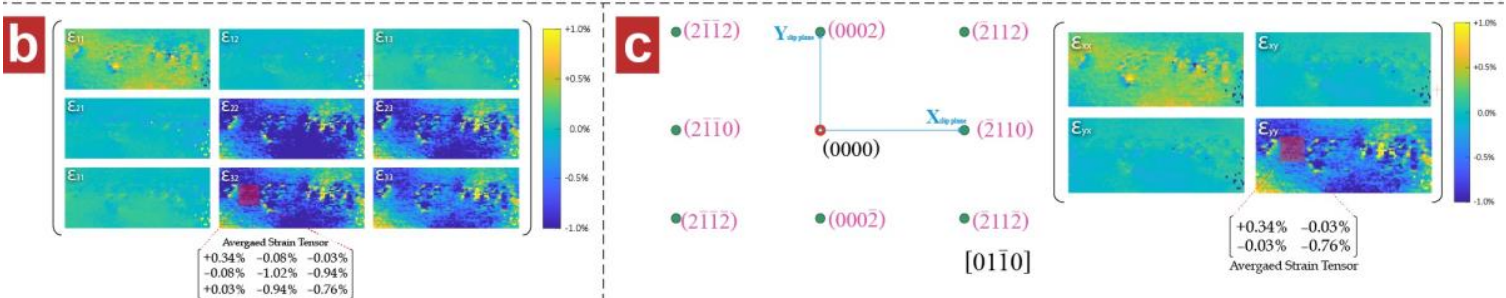

Figure 2. The local strain maps and reconstructed strain tensors; (a), The 2D strain maps of the slip band from 5 different orientations; (b), extracted full strain tensor of the area in a global Cartesian system; (c), The projected 2D strain tensor of [01-10] orientation. 


\section{References}

[1] M.J. Blackburn and J.C. Williams, Trans. ASM 62 (1969), p. 398-409.

[2] Conrad, H., Scripta Metallurgica 7.5 (1973), p. 509-512.

[3] Ding, Jun, et al., PNAS 115.36 (2018): 8919-8924.

[4] Namboodhiri, T.K.G., McMahon, C.J. and Herman, H., Metallurgical Transactions, 4(5) (1973), p.1323-1331.

[5] Fitzner, Arnas, et al., Acta Materialia 103 (2016), p. 341-351.

[6] Neeraj, T., and M. J. Mills, Philosophical Magazine A 82.4 (2002), p. 779-802.

[7] Zhang, Ruopeng, et al., Science advances 5.12 (2019): eaax2799.

[8] Zhang, Ruopeng, et al., arXiv preprint arXiv:1912.05610 (2019).

[9] Neeraj, T., and M. J. Mills, Philosophical Magazine A 82.4 (2002), p. 779-802.

[10] Van de Walle, A., and M. Asta, Metallurgical and Materials Transactions A 33.3 (2002), p. 735-741.

[11] Ozdol, V. B., et al., Applied Physics Letters 106.25 (2015): 253107.

[12] Pekin, Thomas C., et al., Scripta Materialia 146 (2018), p. 87-90.

[13] Zeltmann, Steven E., et al., Ultramicroscopy 209 (2020): 112890.

[14] We gratefully acknowledge funding from the US Office of Naval Research under Grant No. N0001412-1-0413. Work at the Molecular Foundry was supported by the Office of Science, Office of Basic Energy Sciences, of the U.S. Department of Energy under Contract No. DE-AC02-05CH11231. 